Thorax (1973), 28, 680.

\title{
Desquamative interstitial pneumonia: relationship to interstitial fibrosis
}

\author{
ARTHUR S. PATCHEFSKY, HAROLD L. ISRAEL, \\ WILLIS S. HOCH, and GLORIA GORDON \\ Departments of Pathology and Medicine, Thomas Jefferson University Hospital
}

\begin{abstract}
Patchefsky, A. S., Israel, H. L., Hoch, W. S., and Gordon, Gloria (1973). Thorax, 28, 680-693. Desquamative interstitial pneumonia: relationship to interstitial fibrosis. The clinical course and radiographic and pathological findings in 14 patients having the histological pattern of desquamative interstitial pneumonia (DIP) have been studied. Four deaths occurred from cardiorespiratory failure and two from other diseases, and one patient has severe pulmonary insufficiency. Seven patients had altered immunological reactivity or arthritis.

Necropsy of three patients, one who died of respiratory insufficiency and two of other diseases, revealed diffuse pulmonary fibrosis with loss of the desquamative features noted on biopsy. One patient had had industrial exposure to tungsten carbide dust and $x$-ray diffraction analysis of the post-mortem lung showed high concentrations of this substance. Ultrastructural observation and direct immunofluorescent staining of one specimen demonstrated fibrin in the alveolar space, a feature usually regarded as distinguishing DIP from chronic fibrosing interstitial pneumonia.

Our clinical and pathological observations suggest that the histological pattern recognized as DIP may be a non-specific reaction to diverse forms of injury, which is not clearly separable from the usual type of diffuse interstitial fibrosis.
\end{abstract}

Liebow, Steer, and Billingsley (1965) described 18 patients with a distinctive type of interstitial pneumonia characterized by little fibrosis of alveolar septa and marked accumulation of large mononuclear cells in the alveolar space. These authors named this condition, descriptively, desquamative interstitial pneumonia (DIP) and considered it quite separate from the usual chronic fibrosing interstitial pneumonias (UIP) by virtue of its distinctive pathological picture and favourable response to steroid therapy. Gaensler, Goff, and Prowse (1966) corroborated these observations in a series of 12 patients and came to a similar conclusion. The specificity of the pathological changes has since been questioned by several investigators who consider DIP to be not a separate entity but an early stage in the evolution of some cases of idiopathic chronic interstitial fibrosis (Scadding and Hinson, 1967 ; Kuisk and Sanchez, 1969; Coates and Watson, 1971 ; Lemire, Bettez, Gelinas, and

Requests for reprints: Arthur S. Patchefsky, M.D., Department of Surgical Pathology, Thomas Jefferson University Hospital, 11th and Walnut Streets, Philadelphia, Pennsylvania 19107, USA
Raymond, 1972 ; Nagaya and Sieker, 1972). In this report we review our experience of patients whose lungs showed the histological pattern of DIP. Seven patients had associated immunological abnormalities or musculoskeletal complaints, and one had tungsten carbide pneumoconiosis, thus suggesting the nonspecificity of the histological picture. Six of the patients have died and necropsy examination of three revealed interstitial fibrosis with loss of the 'desquamative' features. The pathological findings of the necropsy tissue are described, as well as ultrastructural observations suggesting pathological similarity between DIP and UIP.

\section{CASE MATERIAL}

A retrospective review of all patients indexed in the Medical Records Department of Thomas Jefferson University Hospital between 1959 and 1969 disclosed nine morphologically characteristic examples of DIP. A diagnosis of DIP has been established in three patients admitted to the hospital since 1969, and two additional cases have been seen in consultation. 
T A B L E

CLINICAL FEATURES

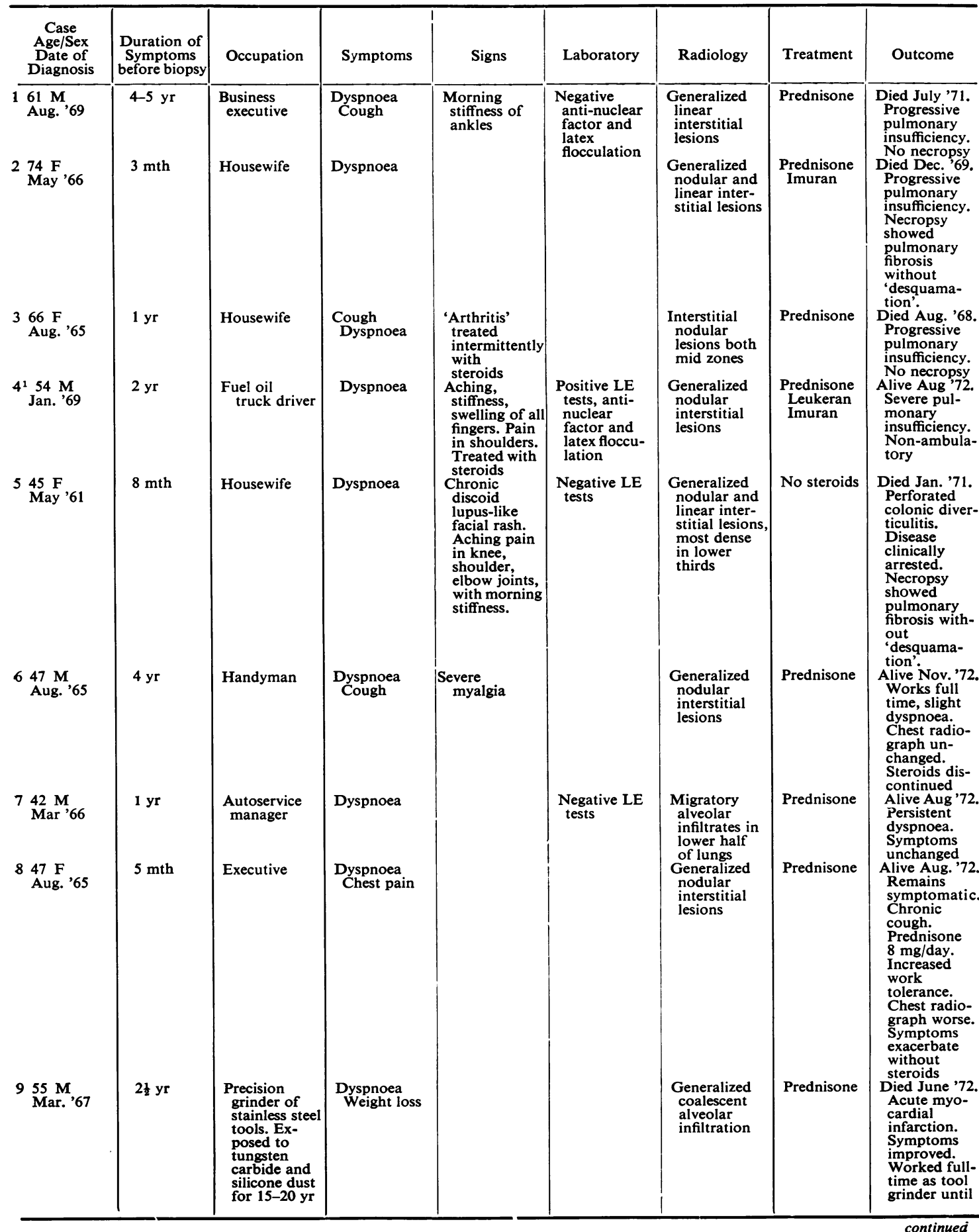


TABLE (continued)

CLINICAL FEATURES

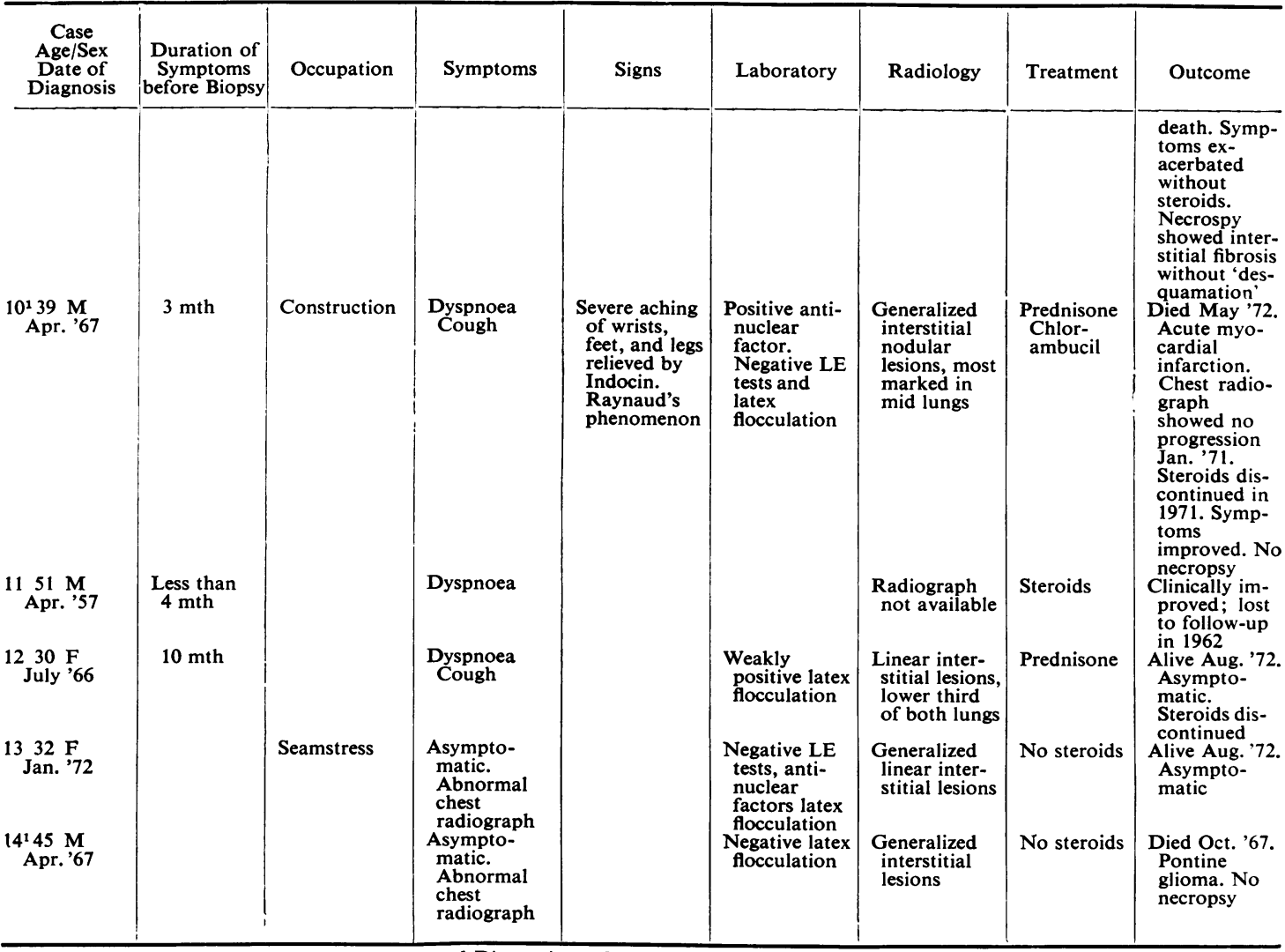

${ }^{1}$ Diagnosis confirmed by Dr. A. Liebow

CLINICAL FINDINGS (Table) The 14 patients ranged in age from 30 to 66 years with the majority in the fifth decade. Eight were men. In 11 patients, dyspnoea on exertion was the predominant symptom. Six patients noted cough, usually nonproductive but occasionally accompanied by small amounts of mucoid sputum. No patient had haemoptysis or pleural effusion. In one patient, symptoms began after an apparent viral upper respiratory tract infection. Three patients were asymptomatic, the only finding being an abnormal chest radiograph. The duration of symptoms before lung biopsy and diagnosis ranged from three months to five years and was longer than one year in six patients.

Physical findings were similar to those previously reported (Liebow et al., 1965 ; Gaensler et al., 1966) and consisted of finely crepitant rales in five patients and clubbing of the fingers in seven patients. Four patients had cyanosis on exertion. Of particular interest was the history of musculoskeletal complaints in six patients (cases 1, 3, 4, 5, 6, and 10). These ranged from morning stiffness to severe arthritis with Raynaud's phenomenon. One patient had a facial rash, consistent clinically with chronic discoid lupus erythematosus, for 15 years before the onset of pulmonary disease (case 5). Of six patients who had serological investigation for circulating auto-antibodies, one had positive antinuclear factor (case 10), one had a weakly positive latex flocculation test (case 12), and one had positive latex flocculation, antinuclear factor, and LE tests (case 4). In the patient with the weakly positive latex flocculation, no signs of arthritis were present. These abnormalities were not noted by Liebow et al. (1965) or Gaensler et al. (1966), but Scadding and Hinson (1967), Corrin and Price (1972), and Leroy (1969) described patients with arthritis or altered immunological activity. Case 9 had been exposed to tungsten carbide dust for approximately 20 years before the onset of symptoms. In view of the recent report of Coates and Watson (1971), suggesting that this substance may be responsible for a disease in some instances histologically identical with DIP, while in others showing interstitial fibrosis, samples of postmortem lung were sent to these workers who kindly performed $x$-ray diffraction analysis of the tissue. 
Their results showed that substantial amounts of tungsten carbide, as well as another unidentified substance, were present.

Pulmonary function tests were performed in eight patients. All showed evidence of restrictive disease with 'diffusion' impairment.

RADIOGRAPHIC APPEARANCE Liebow et al. (1965) described a characteristic radiographic pattern of DIP. Fourteen of their patients had radiographs showing a bilateral fine 'ground glass' reticulated density predominantly in the lower lobes spreading out from the hilum with little evidence of changes in the other portions of the lung. This was thought to be characteristic but not specific for DIP. Three of their patients had normal radiographs of the chest. Gaensler et al. (1966) observed a similar pattern in the majority of their cases. Other reports (Klocke et al., 1967; Kuisk and Sanchez, 1969; Gould, Gleason, and Winterscheid, 1971; McNary and Gaensler, 1971 ; Tushan, Zawadzki, Vassallo, and Robin, 1971) have shown a more diffuse pattern of pulmonary involvement. Review of radiographs of the chest of 13 patients in the present series showed generalized pulmonary disease in 11 patients. Disease was confined to the lower lung fields in only two. The lesions appeared nodular and linear in most instances, while in two patients an alveolar pattern was produced. In no instance was a groundglass appearance noted. Diagnostic possibilities enumerated by the radiologist included sarcoidosis, pneumoconiosis, vascular congestion, and pulmonary fibrosis.

TREATMENT AND OUTCOME Corticosteroids have been widely used in the treatment of DIP. Eleven patients in our series received these drugs. Three patients died of pulmonary insufficiency and one has shown marked progression of disease despite therapy. Six patients given steroids showed symptomatic improvement. Of these, one (case 12) is now free of symptoms and steroids have been withdrawn. Steroids were discontinued in cases 6 and 10, after improvement of symptoms, but the radiograph of the chest remained unchanged. In two patients, symptoms recurred when steroids were withdrawn. In one of them (case 8), the radiographs show continued progression. Case 11 showed improvement after five years but has been lost to follow-up. Two patients died of intercurrent disease after clinical improvement (cases 5 and 9). One patient's symptoms remain unchanged despite steroid therapy (case 7).

Three patients were given immunosuppressive drugs in addition to steroids. One died of the disease, one has progressive pulmonary disability, and one patient improved.

Three patients did not receive corticosteroids. One died of a concurrent pontine glioma, one has remained asymptomatic, with no change in the linear infiltration apparent on serial radiographs, and one patient with predominantly arthritic symptoms did not receive steroids because of intolerance (case 6).

In the reports of Liebow et al. (1965) and Gaensler et al. (1966), the course of DIP appeared benign when compared to the usual form of chronic interstitial pulmonary fibrosis. Only one patient in each of these two series died, one postoperatively and one after 10 years of disease. Two patients in each series had a progressively downhill course. The course has been less favourable in our patients. Three patients are dead of cardiorespiratory failure and one has severe pulmonary insufficiency. Four patients died of intercurrent disease, one of a concurrent pontine glioma, one of perforated colonic diverticulitis, and two of myocardial infarction.

PATHOLOGY As in other reports, the striking feature in our patients' lungs was the presence in the alveolar spaces of numerous large, eosinophilic mononuclear cells. Similar cells were attached to the alveolar septa. It was postulated that these alveolar lining cells which desquamated into the alveolar space consisted predominantly of type II or granular pneumocytes, but since the initial description based on light microscopic observation, several lungs studied with the electron microscope have shown the intra-alveolar cells to consist of macrophages as well as granular pneumocytes, the latter cells lining the alveoli (Brewer, Heath, and Asquith, 1969; Shortland, Darke, and Crane, 1969 ; Farr, Harley, and Hennigar, 1970). The origin of the macrophages is still debated; some authors claim origin from the alveolar septal cells while accumulating evidence suggests origin from the bone marrow (Bowden and Adamson, 1972). Therefore, the term 'desquamative' may not be a correct description of the origin of all the intra-alveolar cells. The reaction was usually spread more or less uniformly throughout the biopsy material, although in some instances it was patchy in distribution. The alveolar septa showed slight to moderate thickening, chiefly by lymphocytes but occasionally by plasma cells, polymorphonuclear leucocytes, and eosinophils. As originally defined, DIP shows little fibrous thickening in the alveolar septa. However, in the original report (Liebow et al., 1965), as well as in subsequent publications, alveolar wall fibrosis has been shown to occur (Gaensler et al., 1966 ; Klocke et al., 1967 ; Gould 
et al., 1971). Thus, while the characteristic findings of large numbers of intra-alveolar cells with little fibrosis morphologically characterizes the disease in its early stages, scarring may supervene, leading to interstitial fibrosis. In our material, eight patients showed mild degrees of fibrosis histologically while still maintaining a predominantly 'desquamative' pattern. Lymphoid nodules located around small bronchioles or in the alveolar septa were common, often with germinal centre formation, and a mild inflammatory infiltration of the pulmonary arterioles without fibrinoid necrosis. A characteristic feature of DIP has been the lack of demonstrable fibrin exudation in the alveolar space and absence of necrosis of intraalveolar cells and alveolar septa. This is in contradistinction to other forms of idiopathic interstitial pneumonia such as that described by Hamman and Rich (1944), and designated as UIP by Liebow, in which fibrin membranes and necrotic cellular debris are regarded as characteristic features of the intra-alveolar exudate (Liebow et al., 1965 ; Liebow, 1968).

Ten of our 14 patients had eosinophilic inclusion bodies in the nuclei of the cells lining the alveoli and those within the alveoli (Patchefsky,
Banner, and Freundlich, 1971). These were characterized by a clear halo around the inclusion body and are therefore suggestive of viral particles. In three patients these were numerous, but in the others they were found only after meticulous search. These bodies were seen in one of Liebow's original series and in one patient reported by Gaensler et al. (1966). Circumstantial evidence suggests that these are not of viral origin as identical structures have been demonstrated in lungs affected by varying diseases. Ultrastructural examination of DIP has failed to show evidence of viral particles (Brewer et al., 1969; Leroy, 1969 ; Shortland et al., 1969 ; Farr et al., 1970 ; Gould et al., 1971; McNary and Gaensler, 1971 ; Corrin and Price, 1972).

Review of the slides from the necropsy tissue from patients 2,5 , and 9 revealed severe pulmonary interstitial fibrosis and honeycomb lung with no residual evidence of the 'desquamative' pattern seen in the initial biopsy (Figs 1 to 6). This was true in the one patient who died of pulmonary insufficiency four years after diagnosis as well as in two patients who had improved clinically 5 and 11 years after diagnosis.

In addition, we were able to examine the lung

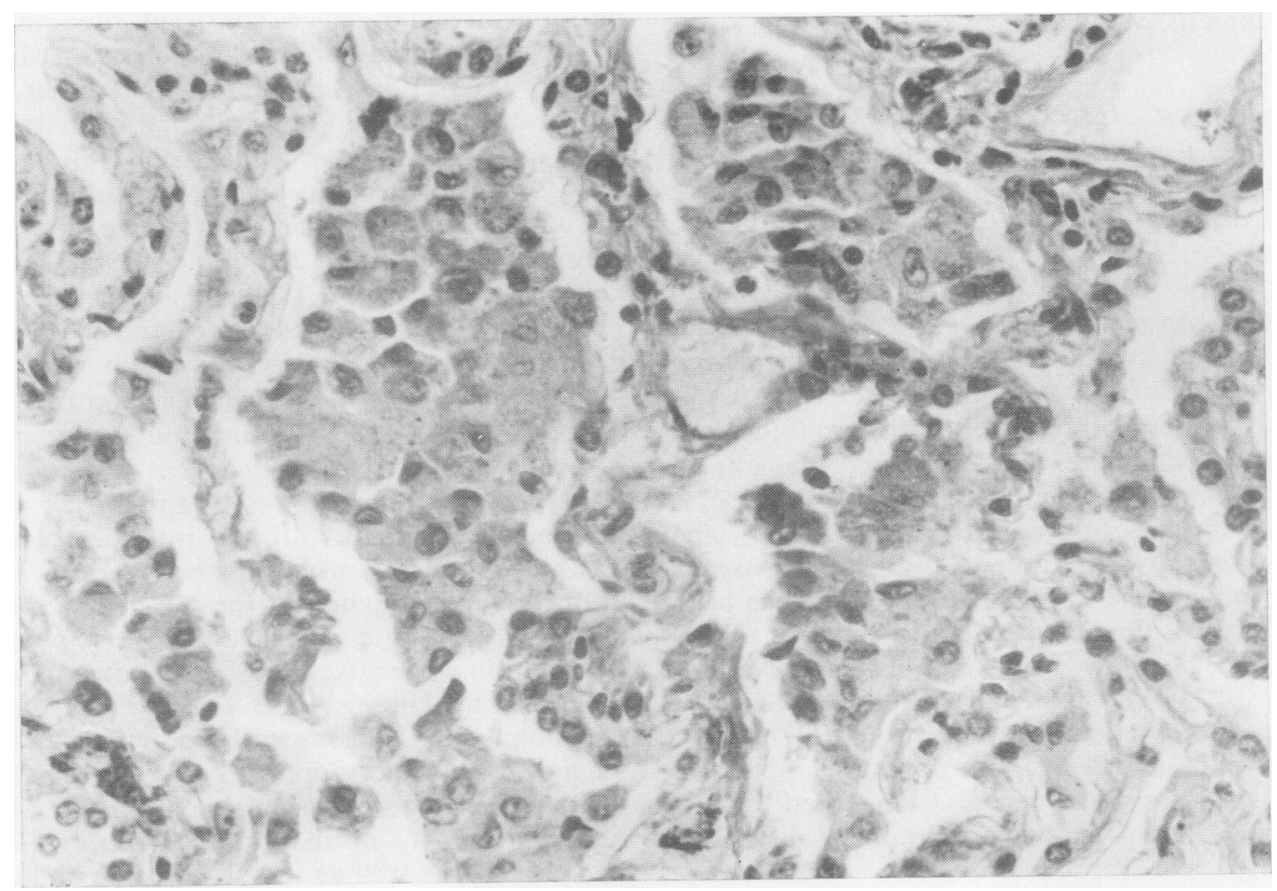

FIG. 1. Case 5. Lung biopsy May 1961. Large numbers of large mononuclear cells in alveoli. Alveolar septa show no thickening. $H$ and $E \times 155$. 


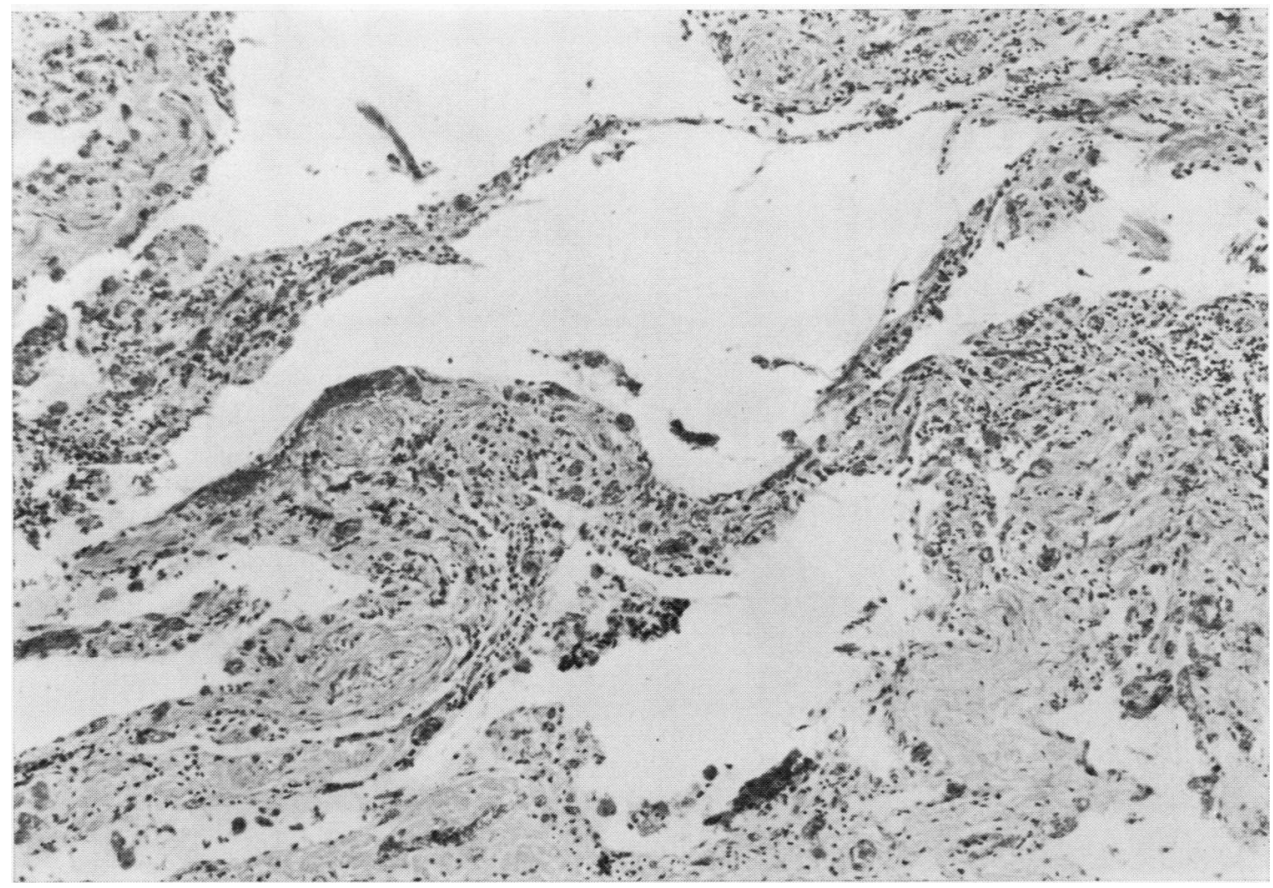

FIG. 2. Case 5. Postmortem lung (January 1971) showing extensive fibrosis and interstitial chronic inflammation with absence of intra-alveolar cells seen in the biopsy. $H$ and $E \times 40$.

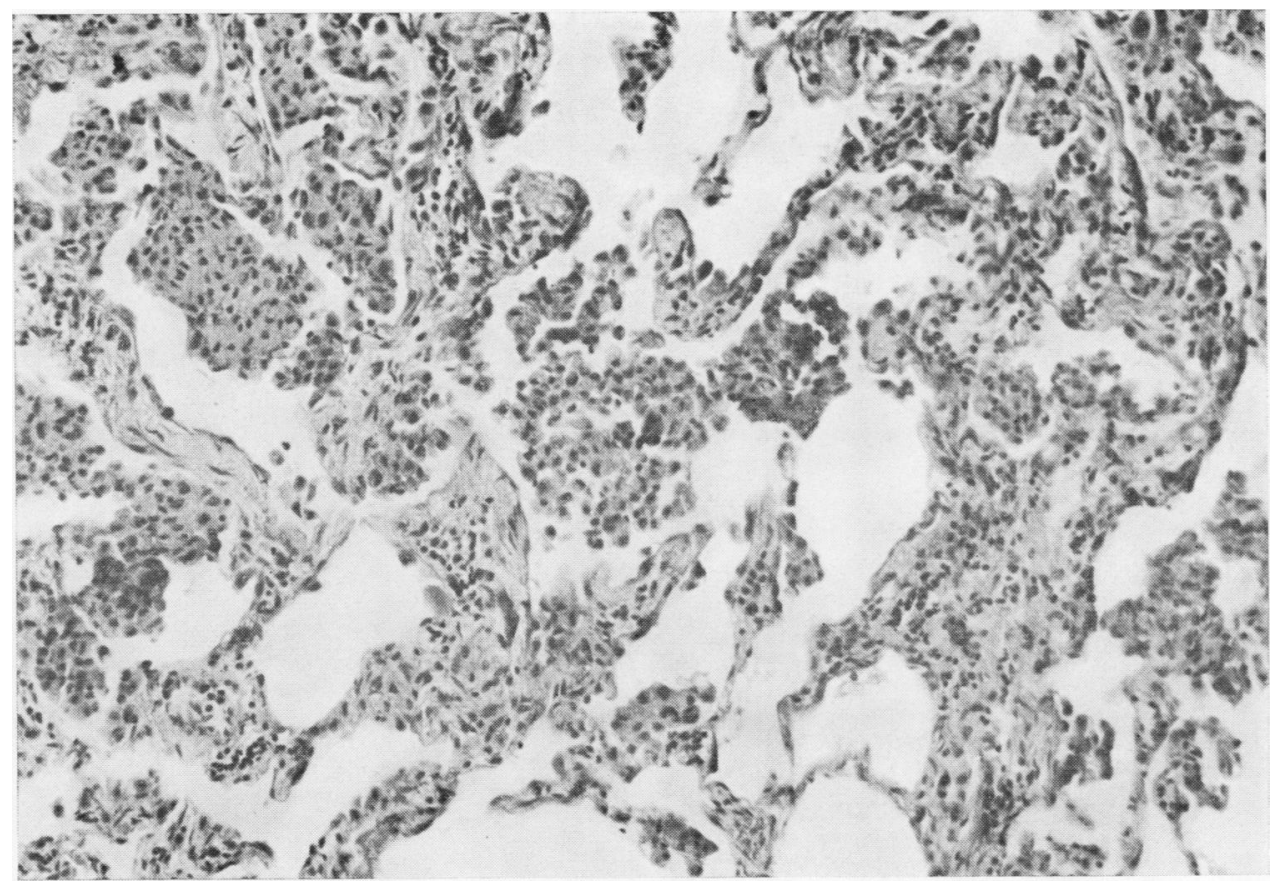

FIG. 3. Case 2. Lung biopsy (May 1966). Mass of intra-alveolar cells suggestive of DIP. Alveolar wall fibrosis present with mild chronic inflammatory infiltration. $H$ and $E \times 155$. 


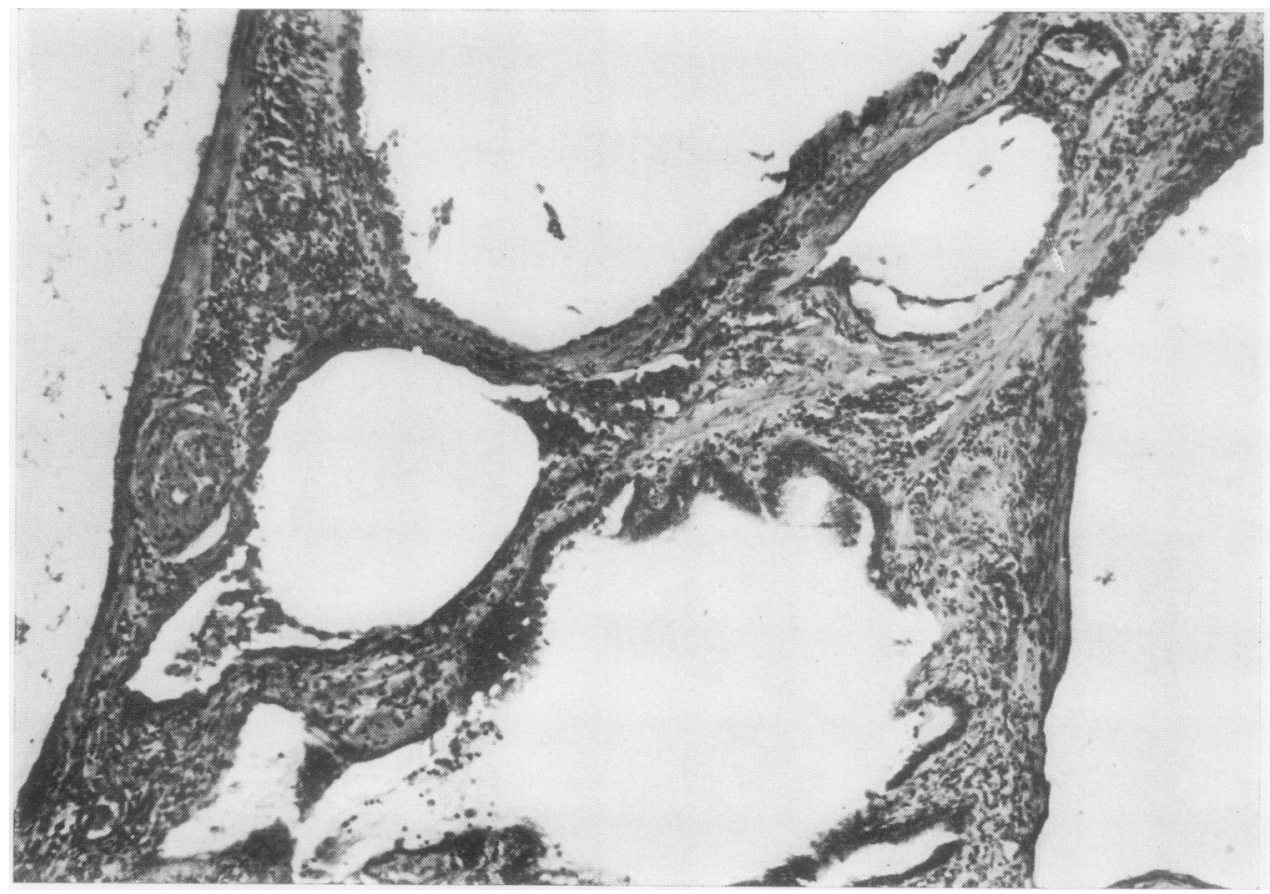

FIG. 4. Case 2. Postmortem lung (December 1969) showing end-stage honeycomb lung. There is severe fibrosis, cystic change, and bronchiolar metaplasia of alveoli. No evidence of the intra-alveolar cells seen in biopsy.

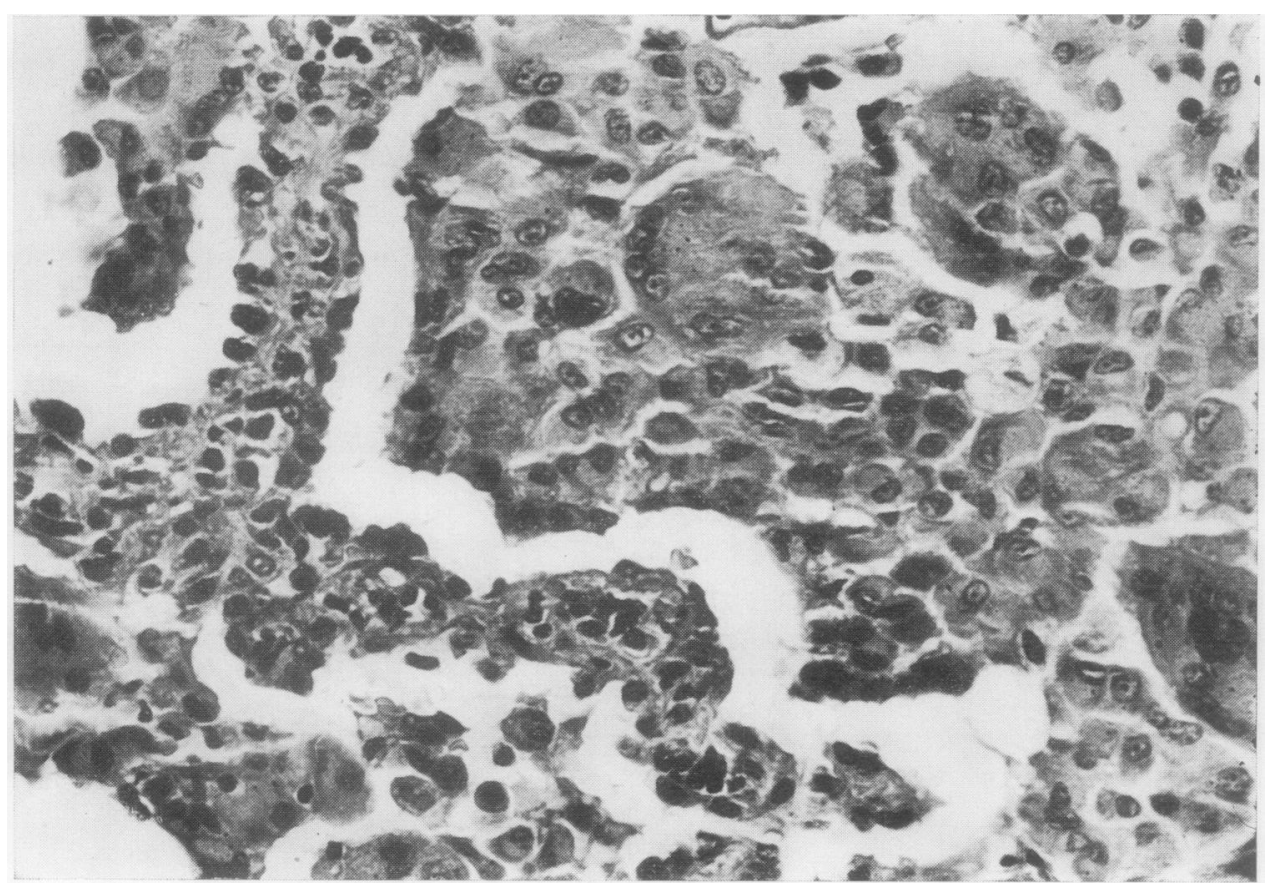

FIG. 5. Case 9. Lung biopsy (March 1967). Sheets of large eosinophilic cells in the alveolar space. Several multinucleated forms are present. The alveolar septa show mild thickening by chronic inflammamatory cells. $H$ and $E \times 155$. 


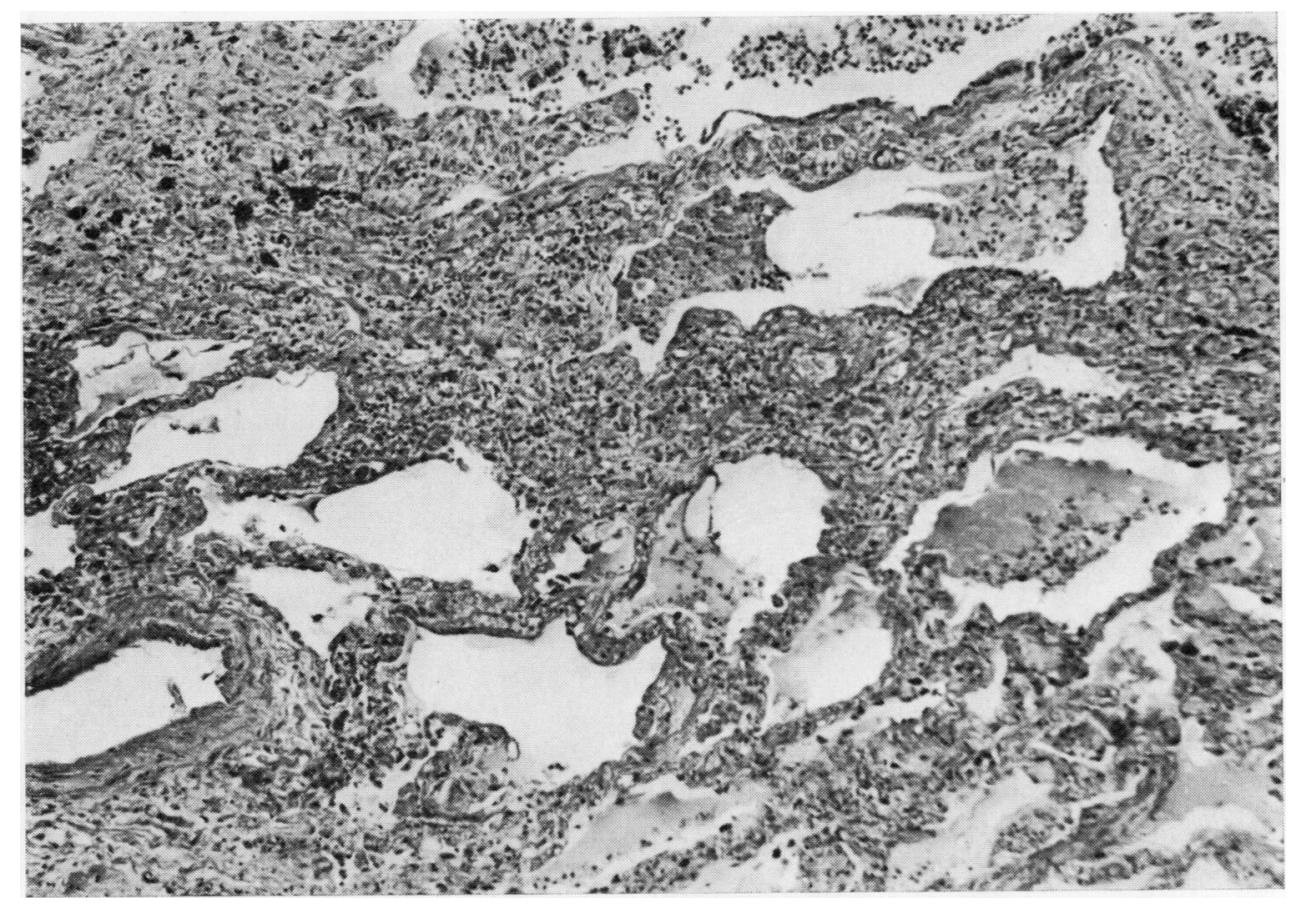

FIG. 6. Case 9. Postmortem lung (June 1972). Marked interstitial fibrosis with chronic interstitial inflammation. Terminal bronchopneumonia. $H$ and $E \times 40$.

biopsy of case 13 by ultrastructural and immunofluorescent techniques (Fig. 7).

On light microscopy of the lung biopsy, scattered patchy areas showed prominence of the alveolar lining cells, alveolar spaces being fiiled with large eosinophilic mononuclear cells (Fig. 8). These stained intensely with PAS and were negative with the Prussian blue reaction. No lymphoid nodules were seen but there was focal lymphocytic infiltration. No fibrin or hyaline membranes were present. The vessels appeared normal. The remaining lung appeared relatively normal except for focal lymphocytic infiltrates in the alveolar septa which were not appreciably thickened. Approximately $20 \%$ of the biopsy was involved by this process. Cultures of the surgical specimen for viruses, mycoplasma, fungi, and mycobacteria were negative.

ULTRASTRUCTURE Tissue for electron microscopy was fixed in $2.5 \%$ phosphate buffered glutaraldehyde for two hours and post-fixed in osmium tetroxide. Sections were embedded in Epon and stained with uranylacetate and lead citrate. Examination was performed with an RCA,

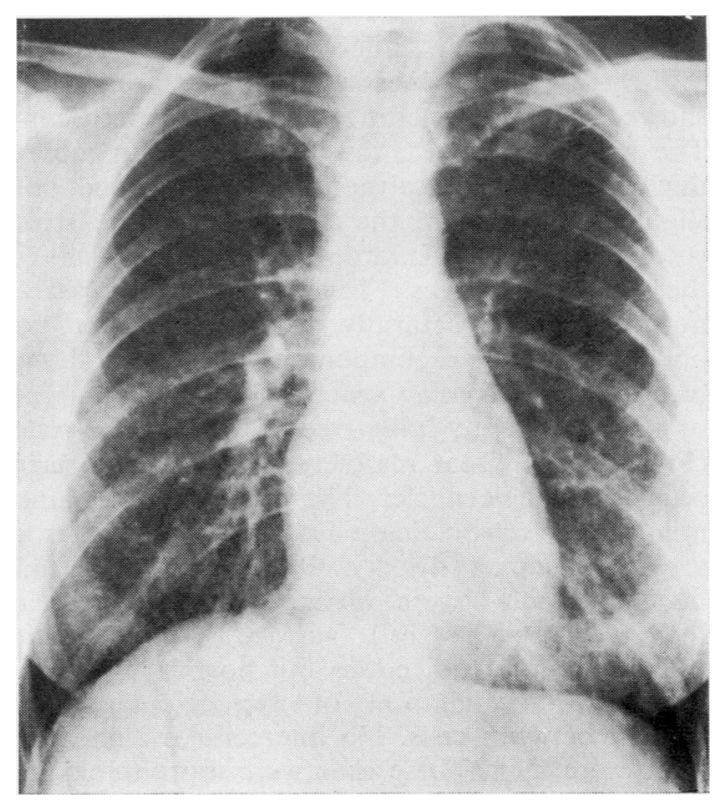

FIG. 7. Case 13. Chest radiograph showing mild diffuse interstitial infiltration. 


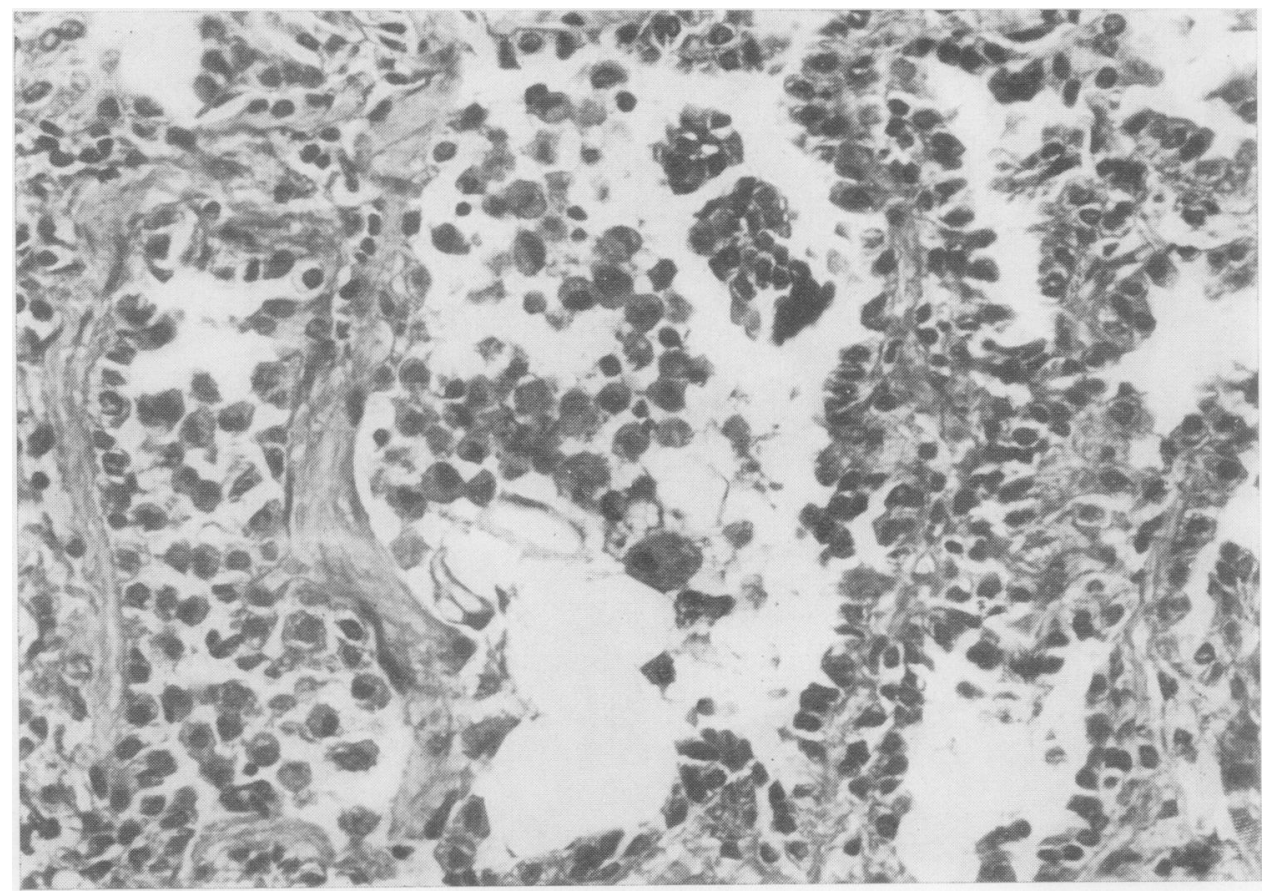

FIG. 8. Case 13. Lung biopsy showing intra-alveolar cellular exudate and mild chronic inflammation in alveolar septa. No haemorrhage, oedema, or fibrin was seen on light microscopy. $H$ and $E \times 155$.

EMU4A electron microscope. The sections arbitrarily selected for ultrastructural study showed minimal alterations by light microscopy. The alveolar septa showed minimal or absent thickening and the alveolar spaces showed only slight prominence of the alveolar lining cells and focal collections of large mononuclear cells in the alveolar spaces. The capillaries appeared normal. Ultrastructurally, slight alteration was observed in each component of the air blood barrier. The alveolar space contained two types of cells apparently lying free in the alveolar space (Fig. 9). The great majority of these were large cells with single nuclei. The cytoplasm contained numerous electron dense lysosome-like bodies of varying electron density. Rare intracytoplasmic lamellar bodies were observed within some of these cells. The cell surface formed blunt pseudopod-like projections but no microvilli, and complex interdigitation of the cytoplasm was present between cells. No intercellular tight junctions were seen. These cells were morphologically suggestive of alveolar macrophages or phagocytic pneumocytes. The presence of rare lamellar bodies within an occasional cell suggests the possibility that some may be transformed granular pneumocytes. The numerous irregular lysosomes and surface pseudopodia suggest phagocytic or digestive function rather than secretory activity of these cells. Other cells much less frequently observed were easily recognized as granular pneumocytes. These contained numerous lamellar bodies and surface microvilli. Cellular debris and free lamellar bodies were found lying within the alveolar space, occasionally adjacent to the alveolar macrophages. Dense linear filamentous structures having the appearance of fibrin were sparsely scattered within the alveolar space usually adjacent to macrophages (Fig. 10A and B). All the cells lining the alveolar space were of two types. The most numerous were the attenuated membranous pneumocytes. Interspersed among these and maintaining contact with the epithelial basement membrane were the granular pneumocytes. These appeared to replace the membranous pneumocytes and were identical with the rare granular pneumocytes in the alveolar space. The number of lamellar bodies within these cells appeared increased. The interstitium contained large dark cells with prominent and multiple 


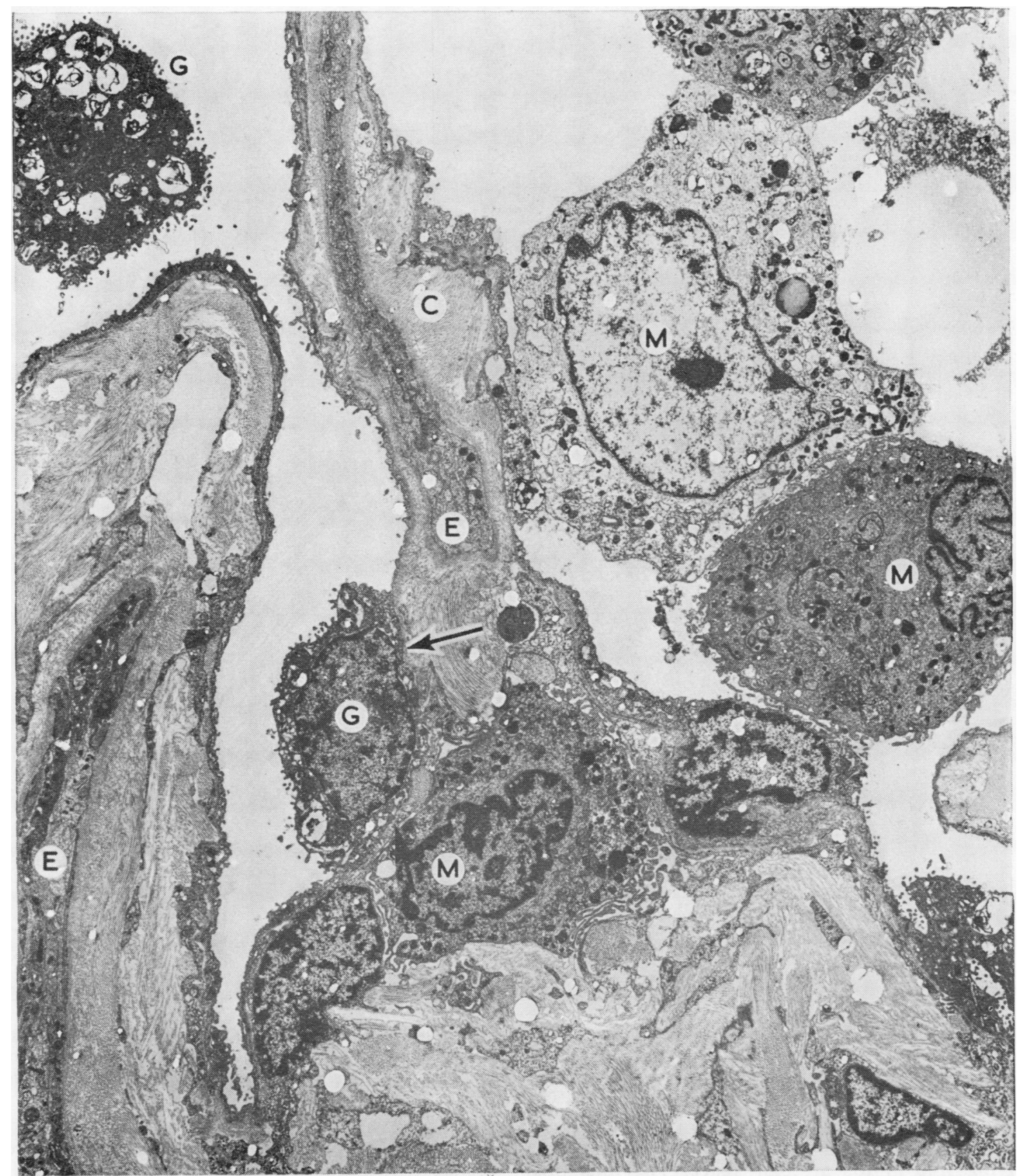

FIG. 9. Attenuated membranous pneumocytes line most of the alveolar space. A free granular pneumocyte (G) as well as macrophages $(M)$ are present in the alveolar space. A macrophage is also present in the interstitium just beneath a granular pneumocyte which is resting on the epithelial basement membrane (arrow). The alveolar wall shows collagen fibres $(C)$ and endothelial swelling $(E) . \times 5,600$. 


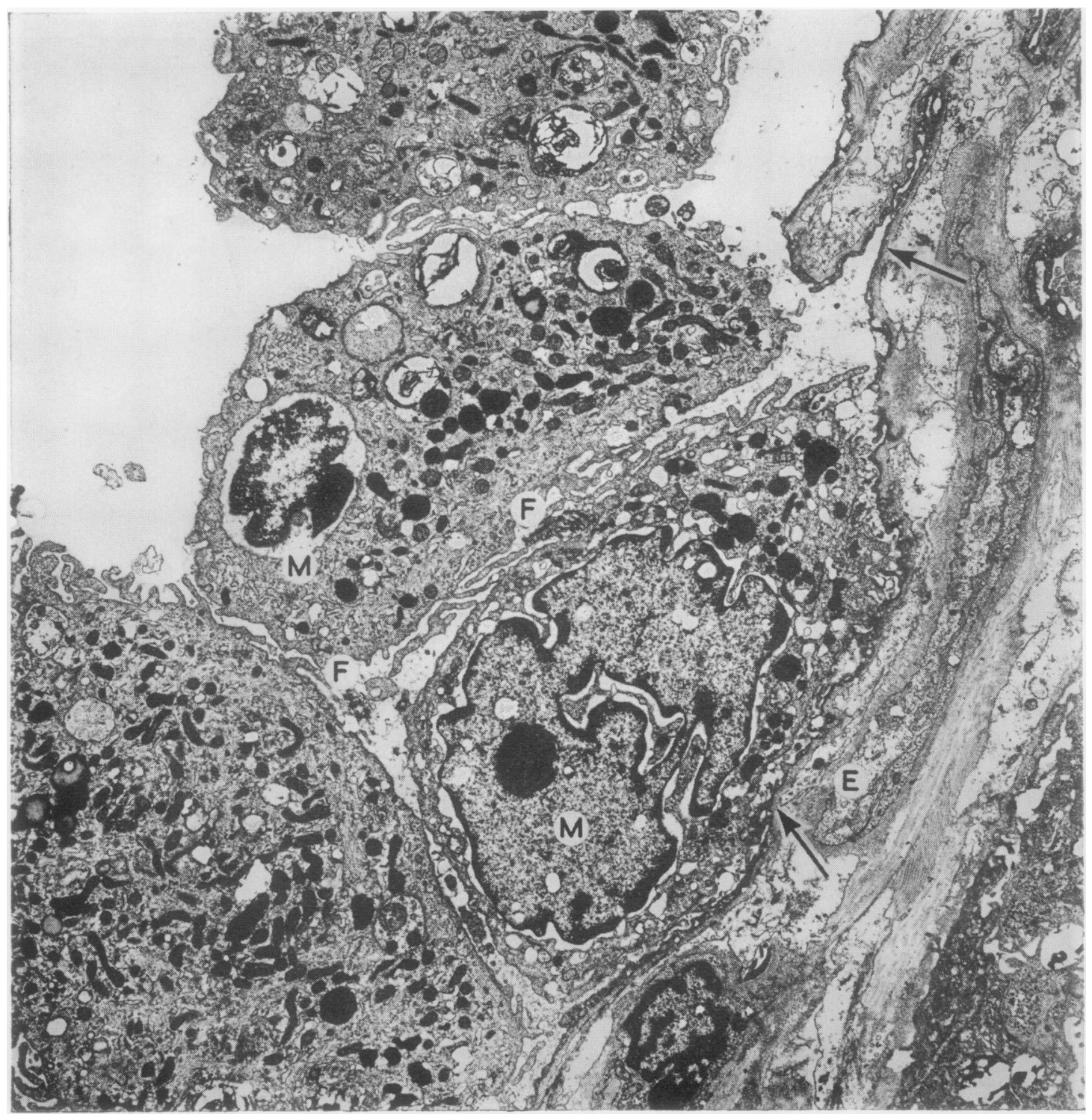

FIG. 10A. Free macrophages $(M)$ are seen in the alveolar space with fibrin-like material $(F)$ present between the cells. $N$ The presence of rare lamellar bodies within these cells suggests the possibility that they may be transformed granular pneumocytes. The attenuated membranous pneumocytes are lying on the epithelial basement membrane (arrows). The endothelial cell cytoplasm $(E)$ appears swollen. $\times 8,500$.

lysosome-like bodies similar to the macrophages lying free in the alveolar space. The interstitial tissue appeared oedematous with focally increased collagen. Occasional mast cells were seen within the interstitium. The capillary endothelial cells were swollen and the cytoplasm sometimes contained vesicles. No abnormalities of the epithelial or capillary basement membranes were $\mathscr{\complement}$ seen. No viral-like particles were identified.

In addition to electron microscopic examination, quick frozen sections were stained by the direct technique, using fluorescein-conjugated goat antihuman IgG, IgM, IgA, complement, fibrinogen, and albumen (Hyland Laboratories). Intense 


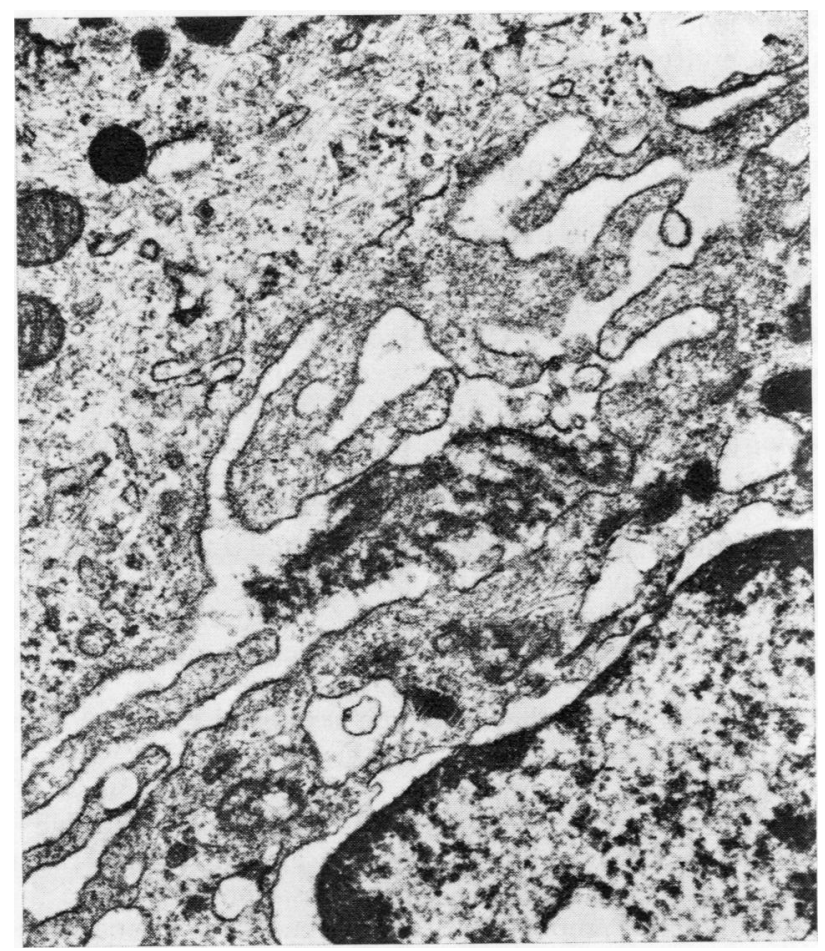

FIG. 10B. Fibrin-like material at higher magnification demonstrating linear characteristics. $\times 28,000$.

staining was seen in the alveolar septa and around intra-alveolar cells with antifibrinogen. Slides stained with the other reagents did not show fluorescence.

\section{DISCUSSION}

Mild changes were present in the case studied by electron microscopy. Nevertheless, examination of these areas afforded an excellent opportunity to observe the early ultrastructural changes of DIP. Our observations in this patient confirm that both macrophages and granular pneumocytes are present in the alveolar space in DIP. The finding of both cell types in a morphologically early lesion suggests that these cells participate simultaneously in the process. The presence of macrophages in the interstitium similar to those in the alveoli suggests that these cells may pass through the alveolar epithelium into the alveolar space. Since no disruption of the basement membrane was present, this may be accomplished by active migration through the epithelium. On the other hand, direct transformation of granular pneumocytes into macrophage-like cells could not be excluded by our studies. There was also evidence of endothelial cell damage and, unlike previous ultrastructural studies, fibrin was demonstrated in the alveoli. The presence of small quantities of fibrin in DIP has not been previously described and suggests that the differences between DIP and other idiopathic interstitial pneumonias or Hamman-Rich disease may be quantitative, the intensity of the alveolar injury determining the form and extent of the morphological changes. Light microscopy may be too insensitive a technique to demonstrate small quantities of this substance.

In earlier studies of DIP we accepted the concept that patients showing this distinctive histological pattern represented a clinical and pathological entity (Patchefsky et al., 1971). Further observation of our patients has caused us to question this hypothesis. The unfavourable outcome in our patients compared to previous studies (Liebow et al., 1965 ; Gaensler et al., 1966) and the opportunity of comparing necropsy material 
with earlier biopsies has led to uncertainty whether DIP represents an entity separable by clinical or radiographic features from the usual form of pulmonary fibrosis.

Although numerous examples of DIP have been reported since 1965, there has not been a sufficiently long follow-up to establish the natural history of this condition. Earlier reports suggested that the outlook of DIP was distinctly better than that of diffuse interstitital fibrosis or UIP and that the disease responded consistently to corticosteroid therapy. The outcome in our patients was less favourable, and the response to steroids inconsistent. To date, three patients have died of respiratory failure from four to seven years after the onset of symptoms and another is now severely incapacitated after five years of illness. At necropsy one of this group showed end-stage honeycomb lung. Necropsies of two other patients who died of intercurrent disease five and 11 years after their clinical disease began showed severe pulmonary fibrosis. Thus, six of the 12 patients who were followed for more than five years after the onset of clinical symptoms showed severe pulmonary damage. These observations support the concept, introduced by Scadding and Hinson (1967), that DIP represents an early stage of chronic interstitial fibrosis. What cannot be answered with certainty is how often patients in whom end-stage fibrosis is found on biopsy started out as DIP. The histological demonstration of fibrin by ultrastructural and immunofluorescent techniques suggests that the pathological differences between DIP and UIP may be merely quantitative. These seemingly different pathological lesions may reflect different intensities or concentrations of the same inciting factors rather than separate pathogenetic mechanisms leading to end-stage pulmonary fibrosis. Since the aetiology of the majority of cases of interstitial fibrosis is unknown, the proof of this hypothesis must await further study.

Scadding and Hinson (1967) found little difference in the clinical course of patients with prominent desquamation of alveolar cells with little alveolar wall thickening compared with those who showed few alveolar cells and established alveolar wall fibrosis. Disease remission was observed in a few patients in both groups. Steroid therapy was able to control the disease only in patients whose biopsy showed many alveolar cells and little fibrosis. However, no difference could be determined in the total duration of the disease from onset of symptoms to death, regardless of the histology of the biopsy when all of their patients were considered. These authors therefore considered DIP as an early phase of 'fibrosing alveolitis'.

The mortality reported in the series of Herbert, Nahmias, Gaensler, and MacMahon (1962) with chronic interstitial fibrosis differs little from that observed in our patients with DIP. These authors observed that death occurred within three years in three patients, while pulmonary insufficiency remained stationary in nine additional patients from two to 15 years after onset. Corticosteroids were ineffective. Scadding (1960) reported that six of 14 patients with fibrosing alveolitis died of pulnonary fibrosis, despite steroid therapy. Four patients who remained alive had prolonged remissions. Other reports are much less favourable (Rubin and Lubliner, 1957 ; Stack, Grant, Irvine, and Moffat, 1965). In Livingstone's study of 45 patients with diffuse interstitial pulmonary fibrosis, 24 patients died of cardiorespiratory failure (Livingstone, Lewis, Reid, and Jefferson (1964). The results of steroid therapy were not impressive in this series.

The prolonged course of patients reported as DIP and the favourable response to steroids would appear to be functions of the amount of fibrosis present at the time of biopsy. Steroids may benefit some patients who have not progressed to the point of significant fibrosis, but once this is established, they appear to have little effect in the course of the disease. As illustrated in our series, steroids may fail to halt the progression of disease, even when 'desquamation' is prominent and fibrosis minimal. Sampling error may have overlooked areas of more advanced scarring in those who progressed. The demonstration of severe fibrosis at necropsy in patients who manifested clinical improvement shows that the disease is often irreversible. Variations in the clinical course of patients with prominent 'desquamation' may be determined by the rate of fibrosis, or the stage at which anti-inflammatory drugs are given.

The aetiology of DIP as well as most other examples of diffuse pulmonary fibrosis remains obscure. The possibility exists of direct or indirect viral injury as a cause, but as yet no viral agents have been cultured from clinical material, nor have viral-like particles been found ultrastructurally. That immunological injury may play a role in the evolution of the disease is suggested by the frequency of serological abnormalities and rheumatoid and lupus-like syndromes observed by us and others (Scadding and Hinson, 1967 ; Leroy, 1969 ; Corrin and Price, 1972). Similar features are seen in chronic interstitial fibrosis, thus obscuring clear separation of the two entities 
on this basis (Turner-Warwick and Doniach, 1965 ; Scadding and Hinson, 1967 ; Nagaya and Sieker, 1972).

It is probable that the histological picture recognized as DIP is a nonspecific reaction pattern by the lung to diverse forms of injury. That certain environmental agents may produce DIP was suggested by Corrin and Price (1972), who found asbestos bodies in one patient. The occurrence of interstitial pneumonia identical with DIP described by Coates and Watson (1971) in tungsten carbide workers was corroborated in one of our patients. It is probable that other causative agents will be discovered that cause alveolar injury giving the histological pattern of DIP. While some patients with this pattern of disease may have a more protracted course than those showing severe interstitial fibrosis, evidence derived from our series indicates that these patients may not form a separate disease entity.

We wish to thank Miss Lois Greenberg and Miss Geraldine Moore for secretarial assistance, and Dr. Leonard Thompkins, Department of Pathology, Misericordia Hospital, Philadelphia, Pennsylvania and Dr. Donald L. Kettering, Pulmonary Disease Section, Mercy Catholic Medical Center, Philadelphia, Pennsylvania for necropsy material on cases 2 and 5 .

\section{REFERENCES}

Bowden, D. H. and Adamson, I. Y. R. (1972). The pulmonary interstitial cell as immediate precursor of the alveolar macrophage. American Journal of Pathology, $68,521$.

Brewer, D. B., Heath, D., and Asquith, P. (1969). Electron microscopy of desquamative interstitial pneumonia. Journal of Pathology, 97, 317.

Coates, E. O. and Watson, J. H. L. (1971). Diffuse interstitial lung disease in tungsten carbide workers. Annals of Internal Medicine, 75, 709.

Corrin, B. and Price, A. B. (1972). Electron microscopic studies in desquamative interstitial pneumonia associated with asbestos. Thorax, 27, 324.

Farr, G. H., Harley, R. A., and Hennigar, G. R. (1970). Desquamative interstitial pneumonia. An electron microscopic study. American Journal of Pathology, 60, 347.

Gaensler, E. A., Goff, A. M., and Prowse, C. M. (1966). Desquamative interstitial pneumonia. New England Journal of Medicine, 274, 113.

Gould, V. E., Gleason, T. H., and Winterscheid, L. C. (1971). Desquamative interstitial pneumonia. Chest, 59, 349.

Hamman, L. and Rich, A. R. (1944). Acute diffuse interstitial fibrosis of the lungs. Bulletin of the Johns Hopkins Hospital, 74, 177.
Herbert, F. A., Nahmias, B. B., Gaensler, E. A., and MacMahon, H. E. (1962). Pathophysiology of interstitial pulmonary fibrosis. Archives of Internal Medicine, 110, 628.

Klocke, R. A., Augerson, W. S., Berman, H. H., Burgos, F. L., and Rivera, R. A. (1967). Desquamative interstitial pneumonia. A disease with a wide clinical spectrum. Annals of Internal Medicine, 66, 498.

Kuisk, H. and Sanchez, J. S. (1969). Desquamative interstitial pneumonia and idiopathic diffuse pulmonary fibrosis. American Journal of Roentgenology, 107, 258.

Lemire, P., Bettez, P., Gelinas, M., and Raymond, G. (1972). Patterns of desquamative interstitial pneumonia (DIP) and diffuse interstitial pulmonary fibrosis (D.I.P.F.). American Jouinal of Roentgenology, 115, 479.

Leroy, E. P. (1969). The blood-air barrier in desquamative interstitial pneumonia (DIP). Virchows Archiv für pathologische Anatomie und Physiologie und für klinische Medizin, 348, 117.

Liebow, A. A. (1968). New concepts and entities in pulmonary disease. International Academy of Pathology Monograph. In The Lung, edited by A. A. Liebow and D. E. Smith, pp. 332-365. Williams and Wilkins, Baltimore, Maryland.

_- Steer, A., and Billingsley, J. G. (1965). Desquamative interstitial pneumonia. American Journal of Medicine, 39, 369.

Livingstone, J. L., Lewis, J. G., Reid, L., and Jefferson, K. E. (1964). Diffuse interstitial pulmonary fibrosis. Quarterly Journal of Medicine, 33, 71.

McNary, W. F. Jr., and Gaensler, E. A. (1971). Intranuclear inclusion bodies in desquamative interstitial pneumonia. Electron-microscopic observations. Annals of Internal Medicine, 74, 404.

Nagaya, H. and Sieker, H. O. (1972). Pathogenetic mechanisms of interstitial pulmonary fibrosis in patients with serum anti-nuclear factor. American Jour nal of Medicine, 52, 51.

Patchefsky, A. S., Banner, M., and Freundlich, I. M. (1971). Desquamative interstitial pneumonia. Significance of intranuclear viral-like inclusion bodies. Annals of Internal Medicine, 74, 322.

Rubin, E. H. and Lubliner, R. (1957). The Hamman-Rich syndrome: review of the literature and analysis of 15 cases. Medicine, 36, 397.

Scadding, J. G. (1960). Chronic diffuse interstitial fibrosis of the lungs. British Medical Journal, 1, 443.

_ and Hinson, K. F. W. (1967). Diffuse fibrosing alveolitis (diffuse interstitial fibrosis of the lungs). Correlation of histology at biopsy with prognosis. Thorax, 22, 291.

Shortland, J. R., Darke, C. S., and Crane, W. A. J. (1969). Electron microscopy of desquamative interstitial pneumonia. Thorax, 24, 192.

Stack, B. H. R., Grant, I. W. B., Irvine, W. J., and Moffat, M. A. J. (1965). Idiopathic diffuse interstitial lung disease: a review of 42 cases. American Review of Respiratory Diseases, 92, 939.

Turner-Warwick, M., and Doniach, D. (1965). Autoantibody studies in interstitial pulmonary fibrosis. Br itish Medical Journal, 1, 886.

Tushan, F. S., Zawadzki, Z. A., Vassallo, C. L., and Robin, E. D. (1971). Serum IgA deficiency in a man with desquamative interstitial pneumonia. American Review of Respiratory Diseases, 103, 264. 